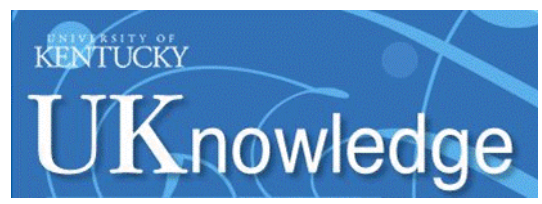

University of Kentucky

UKnowledge

3-16-2015

\title{
Photophysics of Organic Semiconductors: From Ensemble to the Single-Molecule Level
}

\author{
Rebecca Grollman \\ Oregon State University \\ Whitney E. B. Shepherd \\ Oregon State University \\ Alexander Robertson \\ Oregon State University \\ Keshab R. Paudel \\ Oregon State University \\ John E. Anthony \\ University of Kentucky, john.anthony@uky.edu
}

See next page for additional authors

Follow this and additional works at: https://uknowledge.uky.edu/chemistry_facpub

Part of the Chemistry Commons

Right click to open a feedback form in a new tab to let us know how this document benefits you.

\section{Repository Citation}

Grollman, Rebecca; Shepherd, Whitney E. B.; Robertson, Alexander; Paudel, Keshab R.; Anthony, John E.; and Ostroverkhova, Oksana, "Photophysics of Organic Semiconductors: From Ensemble to the SingleMolecule Level" (2015). Chemistry Faculty Publications. 45.

https://uknowledge.uky.edu/chemistry_facpub/45

This Article is brought to you for free and open access by the Chemistry at UKnowledge. It has been accepted for inclusion in Chemistry Faculty Publications by an authorized administrator of UKnowledge. For more information, please contact UKnowledge@lsv.uky.edu. 


\section{Photophysics of Organic Semiconductors: From Ensemble to the Single-Molecule Level}

Digital Object Identifier (DOI)

http://dx.doi.org/10.1117/12.2079755

\section{Notes/Citation Information}

Published in Proceedings of SPIE, v. 9360, Organic Photonic Materials and Devices XVII, article 93600V, p. 1-8.

Copyright 2015 Society of Photo Optical Instrumentation Engineers. One print or electronic copy may be made for personal use only. Systematic reproduction and distribution, duplication of any material in this paper for a fee or for commercial purposes, or modification of the content of the paper are prohibited.

Rebecca R. Grollman, Whitney E. B. Shepherd, Alexander Robertson, Keshab R. Paudel, John E. Anthony, Oksana Ostroverkhova. "Photophysics of organic semiconductors: from ensemble to the single-molecule level", Proceedings of SPIE 9360, Organic Photonic Materials and Devices XVII, $93600 \mathrm{~V}$ (March 16, 2015).

http://dx.doi.org/10.1117/12.2079755

\section{Authors}

Rebecca Grollman, Whitney E. B. Shepherd, Alexander Robertson, Keshab R. Paudel, John E. Anthony, and Oksana Ostroverkhova 


\title{
Photophysics of organic semiconductors: from ensemble to the single-molecule level
}

\author{
Rebecca R. Grollman, ${ }^{a}$ Whitney E. B. Shepherd, ${ }^{a}$ Alexander Robertson, ${ }^{a}$ Keshab R. Paudel, ${ }^{a}$ \\ John E. Anthony, ${ }^{b}$ and Oksana Ostroverkhova ${ }^{a}$ \\ ${ }^{a}$ Department of Physics, Oregon State University, Corvallis, OR 97331 \\ ${ }^{b}$ Department of Chemistry, University of Kentucky, Lexington, KY 40506
}

\begin{abstract}
We present photophysical properties of functionalized anthradithiophene (ADT) and pentacene (Pn) derivatives, as well as charge and energy transfer properties of donor-acceptor (D/A) pairs of these derivatives. All molecules studied were fluorescent and photostable enough to be imaged on the single-molecule level in a variety of polymeric and in a functionalized benzothiophene (BTBTB) crystalline host using room-temperature widefield epifluorescence microscopy. Flexibility of functionalization of both guest (ADT, Pn) and host (BTBTB or polymer) molecules can be used for systematic studies of nanoscale morphology and photophysics of D/A organic semiconductor bulk heterojunctions, as well as in applications relying on FRET, using single-molecule fluorescence microscopy.
\end{abstract}

Keywords: Single molecule fluorescence, organic semiconductors, FRET, charge transfer, photobleaching

\section{INTRODUCTION}

Organic semiconductors have attracted attention due to their applications in light-emitting diodes, solar cells, thin film transistors, and many others and due to their low cost, easy fabrication, and tunable properties. ${ }^{1}$ Most successful organic materials for applications that rely on charge carrier photogeneration (e.g. solar cells) involve donor-acceptor (D/A) bulk heterojunctions (BHJs), properties of which are determined by photoinduced D/A interactions. Examples of these include charge transfer (CT) and Förster resonant energy transfer (FRET), which depend on the photophysical properties of the donor and acceptor molecules, as well as on the nanoscale morphology and local nanoenvironment. ${ }^{2-8}$

Single-molecule fluorescence spectroscopy (SMFS) has been extensively utilized for probing nanoscale interactions and local nanoenviroment in polymers, crystalline solids, and biological systems. ${ }^{9-11}$ Single molecules (SM) have also been used as probes of conduction and of exciton dynamics in organic semiconductors. ${ }^{12-15}$ However, because of stringent requirements for the good fluorophores for the SMFS (which include high fluorescence quantum yield and high photostability), studies of local nanoenvironment and its effect on photophysics of organic semiconductors have been limited to a handful of materials. In this paper, we discuss high-performance functionalized organic semiconductor materials which are suitable for systematic studies of photophysics which includes charge and energy transfer, both on the macroscopic (device) level and at nanoscales, down to the SM level.

\section{EXPERIMENTAL}

\subsection{Materials and Sample Preparation}

In our studies, we used functionalized anthradithiophene (ADT) derivatives with (triethylsilyl)ethynyl (TES) and (triisopropylsilyl)ethynyl (TIPS) side groups (ADT-TES-F and ADT-TIPS-CN, as shown in Fig. 1a) and pentacene (Pn) derivatives with TIPS, NODIPS ((n-octyldiisopropylsilyl)ethynyl), or TCHS ((tricyclohexylsilyl)ethynyl) side groups (Fig. 1b,d) as donors and acceptors for thin-film studies as well as fluorescent guests embedded in a solid host for SMFS. The ADT-TES-F derivative is a high-performance organic semiconductor which exhibits TFT charge carrier mobilities of over $1.5 \mathrm{~cm}^{2} /(V s)$ in spin-cast films, ${ }^{16}$ high photoconductive gains, and fast charge carrier photogeneration. ${ }^{17,18}$ Both ADT-TIPS-CN and Pn-R-F8 (R=TIPS, NODIPS, TCHS) derivatives have been used as acceptors in D/A BHJs with polymer or ADT-TES-F donors. ${ }^{2,4,5,19-21}$ 

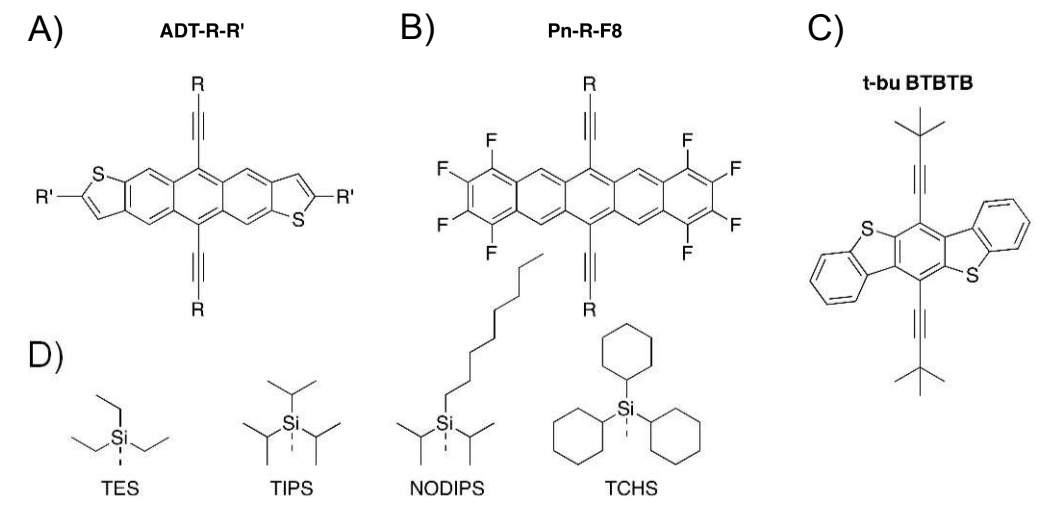

NODIPS

Figure 1. A) ADT-R-R' where R is a side group and R' is an end group (either F or CN), B) Pn-R-F8 where R is a side group, C) t-bu BTBTB, D) Variety of side groups including TES, TIPS, NODIPS, and TCHS.

Pristine films of ADT-R-R' and Pn-R-F8 derivatives exhibit $\pi$-stacking, ${ }^{2}$ where R-groups control the packing motif. $^{6}$

As host matrices, we chose functionalized benzothiophene derivative t-bu BTBTB (6,12-bis[2-(t-butyl)ethynyl]benzo[1,2-b:4,5-b']bis(1)benzothiophene, as shown in Fig. 1c), poly(methyl)methacrylate (PMMA), poly(9-vinylcarbazole) (PVK), and polystyrene (PS). The optical absorption of the t-bu BTBTB derivative, as well as of PMMA, PVK, and PS, is in the UV region, ${ }^{22}$ which enables their utility as non-fluorescent hosts for SM imaging at $532 \mathrm{~nm}$ and $633 \mathrm{~nm}$. The t-bu BTBTB derivative forms spin-cast polycrystalline films with a dominant (01-1)crystallite orientation ${ }^{23}$ and exhibits photoconductivity under UV excitation. ${ }^{22}$

Several types of spin-cast films were prepared. For SMFS, ADT-R-R' or Pn-R-F8 guest molecules were added at $10^{-10} \mathrm{M}$ concentrations to a $1 \%$ wt solution of PMMA (75,000 m.w., Polysciences, Inc.), PVK (1,100,000 m.w., Aldrich), or PS (280,000 m.w., Aldrich) in toluene. Guest molecules at $10^{-12} \mathrm{M}$ concentrations were added to a $10^{-2} \mathrm{M}$ concentration of t-bu BTBTB in tetrahydrofuran (THF). Glass coverslips were soaked in a detergent and water solution overnight. They were then sonicated for 40 minutes in the detergent/water solution, rinsed thoroughly with deionized water, and dried under $N_{2}$. After confirming that glass coverslips, solvent, and host matrices were clean in the single-molecule imaging configuration, guest-host samples were spin-cast onto slides for 50 seconds at $3000 \mathrm{rpm}$ for polymer samples and at $2000 \mathrm{rpm}$ for t-bu BTBTB samples.

Spin-cast films for fluorescence lifetime, quantum yield (QY), and photobleaching QY measurements were prepared with higher guest concentrations ("bulk" samples). For QYs, samples were prepared from $10^{-4} \mathrm{M}$ stock solutions of guest molecules in toluene and either $13 \%$ wt solution of PMMA in toluene or $10^{-2} \mathrm{M}$ solution of t-bu BTBTB in THF. The concentrations of guest molecules were chosen to achieve the average spacing between guest molecules of 5 to $7 \mathrm{~nm}$, to ensure minimal guest-guest interaction. ${ }^{24}$ Spin-cast films for FRET measurements were also prepared with 50/50 mixtures of ADT-TES-F and Pn-F-F8 in PMMA to achieve an average spacing between donor and acceptor molecules of $2 \mathrm{~nm}$. Donor- and acceptor-only samples were also prepared with the same spacing. All "bulk" samples were spin cast at $600 \mathrm{rpm}$ for 50 seconds.

\subsection{Photobleaching Quantum Yield}

Details of measurements of fluorescence QY and lifetimes have been reported in our previous publications. ${ }^{2,3,5}$

Photobleaching experiments were done with "bulk" samples in the PMMA host under $633 \mathrm{~nm}$ (Pn-R-F8) or $532 \mathrm{~nm}$ (ADT-R-R') excitation with light intensity $\left(I_{\lambda}\right)$ of $28 \mathrm{~W} / \mathrm{cm}^{2}$. Similarly, samples in the t-bu BTBTB host were excited with an intensity of $185 \mathrm{~W} / \mathrm{cm}^{2}$. Fluorescence spectra were collected as a function of time with a fiber coupled spectrometer (Ocean Optics USB2000) and integrated over all emission wavelengths for each time frame, ranging from 0.5 to 2 seconds. Most experiments were performed in air, with selected samples measured both in air and in vacuum at $10^{-5}$ Torr using a micro-cryostat (Janis STC-500) at excitation with light intensity of $0.28 \mathrm{~W} / \mathrm{cm}^{2}$. The photobleaching dynamics of PMMA samples were fit with a bi-exponential $\left(a_{1} \exp \left[-t / \tau_{B 1}\right]+\right.$ $\left.a_{2} \exp \left[-t / \tau_{B 2}\right]\right)$ function. The time constant $\left(\tau_{B}\right)$ is a weighted average of the two time constants $\tau_{B 1}$ and 


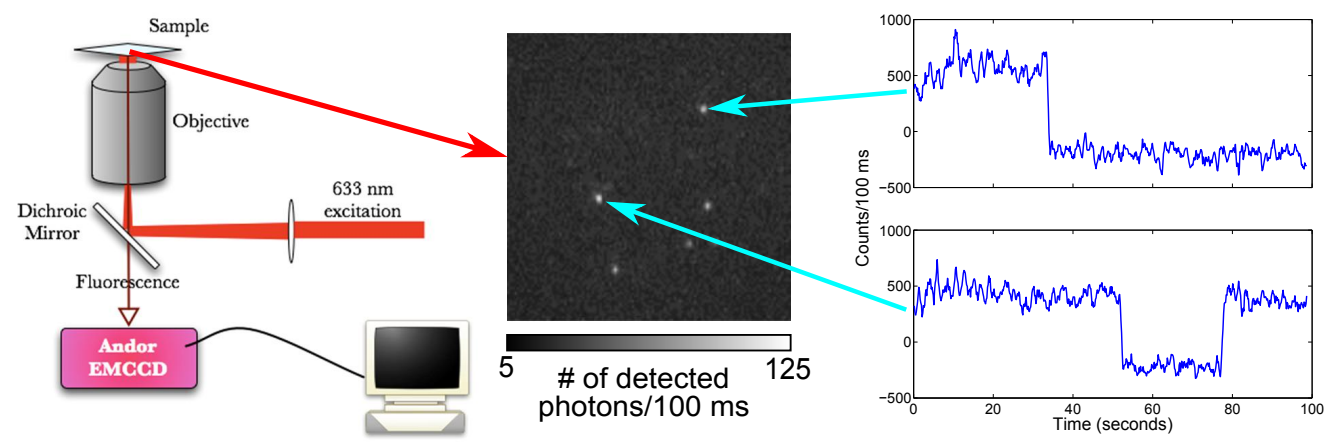

Figure 2. Left: Schematic of the experimental set up for single molecule fluorescence spectroscopy. The excitation was $633 \mathrm{~nm}(532 \mathrm{~nm})$ in the case of Pn-R-F8 (ADT-R-R') derivatives. Middle: Image of single molecules of Pn-TCHS-F8 in PMMA. Right: Fluorescence time trajectories for the two molecules. The top represents a molecule that photobleaches. The bottom represents a molecule that blinks.

$\tau_{B 2}$. The photobleaching dynamics of t-bu BTBTB samples were fit with a single exponential. Photobleaching quantum yield $\Phi_{B}$ was calculated based on Eq. 1

$$
\Phi_{B}=\frac{1}{\tau_{B} \sigma_{\lambda} I_{\lambda} \frac{\lambda}{h c}},
$$

where $\sigma_{\lambda}$ is the absorption cross-section at the wavelength of excitation $(\lambda), h$ is the Planck constant, and $c$ is the speed of light. The photobleaching quantum yield was also used along with the fluorescence quantum yield $\left(\Phi_{F}\right)$ to calculate the total number of photons emitted by the molecule $N_{\text {tot }}$ using

$$
N_{t o t}=\frac{\Phi_{F}}{\Phi_{B}}
$$

\subsection{Single Molecule Spectroscopy}

Single molecule imaging of Pn-R-F8 derivatives and ADT-TIPS-CN was done under circularly polarized 633 $\mathrm{nm}$ or $532 \mathrm{~nm}$, respectively, wide-field illumination using an Olympus IX-71 inverted microscope with a 100x UPlanSApo (NA 1.4) oil objective and an Andor iXon EMCCD (DU-897) detector (see Fig. 2). Collection efficiency $\eta_{\text {coll }}$ was determined by $\eta_{\text {coll }}=\eta_{Q} T_{a n g} T_{o p t} T_{\text {filt }}$ where $\eta_{Q}$ is the quantum efficiency of the camera (ranging from $85 \%$ to $94 \%$ depending on the emission from the molecule), $T_{a n g}$ is the angular collection factor (34\% based on Ha et al. ${ }^{25}$ and Lord et al. $\left.{ }^{26}\right), T_{o p t}$ is the collection factor through microscope optics $(62 \%$ to $68 \%$ ), and $T_{\text {filt }}$ is the transmission through the dichroic and emission filters (21\% to $\left.65 \%\right) .{ }^{26}$ We estimated our collection efficiency to be about $4.6 \%$ for ADT-TES-F, $12.0 \%$ for ADT-TIPS-CN and $10.6 \%$ for Pn-R-F8.

Multiple videos of 600 frames (100 ms/frame) were recorded of each sample in a variety of host matrices. Custom MATLAB scripts then located potential fluorophores and displayed their time trace. Each time trace was reviewed to confirm digital blinking or photobleaching. "On" and "off" counts were calculated based on anything above or below a threshold of three standard deviations above the "off" level, unless stated otherwise. The total number of detected photon $N_{\text {tot }}$ was determined again using data collected from the single molecule time traces. The total number of detected photons was calculated by integrating the each time trace (without the background) over it's lifetime. Histograms of the number of detected photons were fit to a single-exponential function $\left(\exp \left[-N / N_{t o t, d e t}\right]\right)$ to determine the number of detected photons $N_{t o t, d e t}$. The number of emitted photons $N_{t o t, e m}$ was calculated by dividing $N_{\text {tot,det }}$ by the collection efficiency $\eta_{\text {coll }}$ defined above. 


\section{RESULTS AND DISCUSSION}

Photophysical properties of functionalized ADT and Pn derivatives under study are summarized in Table 1. High fluorescence QYs were observed in toluene; these were further improved when the molecules were incorporated into a solid host. The photobleaching QY, also shown in Table 1, is the probability that a molecule would photobleach upon absorption of a photon. Partial fluorination of the molecular backbone in functionalized $\mathrm{ADT}, \mathrm{Pn}$, or hexacene derivatives has been realized as an effective means to improve stability of the molecule against oxidation due to substitution of the most reactive site and electronic structure changes induced by electron-negative fluorine substituent. For example, under the same illumination conditions, in air, Pn-R-F8 molecules dispersed in PMMA were considerably more photostable than their counterparts (Pn-R) with no fluorine substitution (Fig. 3a). The residual photodegradation of ADT-R-R and Pn-R-F8 derivatives dispersed in PMMA observed in air was significantly reduced in vacuum (Fig. 3b), which confirms that photobleaching of these molecules is largely due to photoinduced reaction with oxygen. In PMMA, the values of $\Phi_{B}$ of $(1-2.5)$ $\times 10^{-6}$ obtained in ADT and Pn derivatives (Table 1) were comparable with those of commonly used SMFS fluorophores (e.g. dicyanomethylenedihydrofuran (DCDHF) derivatives), in a similar environment. ${ }^{26,27}$ The photobleaching QYs for the Pn-R-F8 molecules embedded in the crystalline t-bu BTBTB host were more than an order of magnitude lower than those in PMMA, depending on the derivative, in part due to a reduced oxygen diffusion in the t-bu BTBTB crystalline environment. High fluorescence QYs and high photostability exhibited by ADT-R-R' and Pn-R-F8 derivatives under study enabled their imaging in a variety of hosts (PMMA, PVK, PS, and t-bu BTBTB) at the single molecule level under wide-field excitation at either $532 \mathrm{~nm}$ (ADT-R-R') or $633 \mathrm{~nm}$ (Pn-R-F8) (Fig.2b). Effects of different host environments on the single-molecule photophysics will be reported elsewhere. ${ }^{23}$

Table 1. Photophysical properties of functionalized organic semiconductors under study.

\begin{tabular}{|l|l|l|l|l|l|l|l|}
\hline Molecule & $\begin{array}{l}\lambda_{a b s}{ }^{[\mathrm{a}]} \\
(\mathbf{n m})\end{array}$ & $\begin{array}{l}\lambda_{e m}{ }^{[\mathrm{b}]} \\
(\mathbf{n m})\end{array}$ & $\begin{array}{l}\Phi_{F}{ }^{[\mathrm{c}]} \\
(\mathbf{P M M A})\end{array}$ & $\begin{array}{l}\tau^{[\mathrm{d}]} \\
(\mathbf{n s})\end{array}$ & $\begin{array}{l}\Phi_{B},{ }^{[\mathrm{e}]} \\
10^{-6}\end{array}$ & $\begin{array}{l}N_{\text {tot }}{ }^{[\mathrm{f}]} \\
10^{5}(\mathbf{b u l k})\end{array}$ & $\begin{array}{l}N_{\text {tot, },{ },{ }^{[\mathrm{g}]}} \\
0^{5}(\mathbf{S M})\end{array}$ \\
\hline ADT-TES-F & 528 & 536 & $0.7(0.9)$ & 9.4 & 1.1 & 8.2 & 7.7 \\
\hline ADT-TIPS-CN & 582 & 590 & $0.76(0.89)$ & 12.7 & 2.5 & 3.6 & 2.4 \\
\hline Pn-TIPS-F8 & 635 & 645 & $0.6(0.8)$ & 9.4 & 1.9 & 4.2 & 4.7 \\
\hline Pn-NODIPS-F8 & 635 & 645 & $0.53(0.67)$ & 6.4 & 1.2 & 5.6 & 3.8 \\
\hline Pn-TCHS-F8 & 637 & 647 & $0.61(0.82)$ & 8.7 & 1.0 & 8.2 & 9.4 \\
\hline
\end{tabular}

${ }^{[a]}$ Wavelengths of lowest energy absorption maximum measured in dilute toluene solutions.

${ }^{[b]}$ Wavelengths of maximal emission measured in dilute toluene solutions.

${ }^{[c]}$ Fluorescence QY in toluene. The values in parenthesis are from bulk samples in PMMA.

[d] Fluorescence lifetime in toluene.

${ }^{[\mathrm{e}]}$ Photobleaching QY of Eq. 1 obtained in bulk samples in PMMA.

[f] Total number of emitted photons calculated using Eq. 2.

[g] Mean number of total emitted photons obtained from single exponential fits to SM histograms.

The total number of photons emitted $N_{\text {tot,em }}$ in single molecule studies were similar to those that were calculated through Eq. 2 from photobleaching-related fluorescence decays measured in "bulk" samples (Fig.3). For example, the SM value $N_{\text {tot,em }}$ of $2.4 \times 10^{5}$ photons emitted compared well to $3.6 \times 10^{5}$ photons from the "bulk" value for ADT-TIPS-CN in PMMA (Table 1). Nevertheless, we tested the robustness of the approach we used in our MATLAB script that selects fluorophores from the video, analyzes SM fluorescence time trajectories such as those in Fig.2, and calculates $N_{\text {tot,em }}$. In particular, we varied the threshold which separates the "on" and "off" states of the molecule (e.g. Pn-TCHS-F8 in PMMA in Fig. 4) and determined the variation in the obtained values of $N_{t o t, e m}$. For Pn-TCHS-F8 molecules in PMMA, this yielded the range of $N_{t o t, e m}$ between $6.9 \times 10^{5}$ and $9.4 \times 10^{5}$ photons, which provides an estimate for the analysis-related error in the $N_{\text {tot,em }}$ values.

It has been previously shown that ADT-TES-F/ADT-TIPS-CN blends exhibit either FRET (with the FRET 

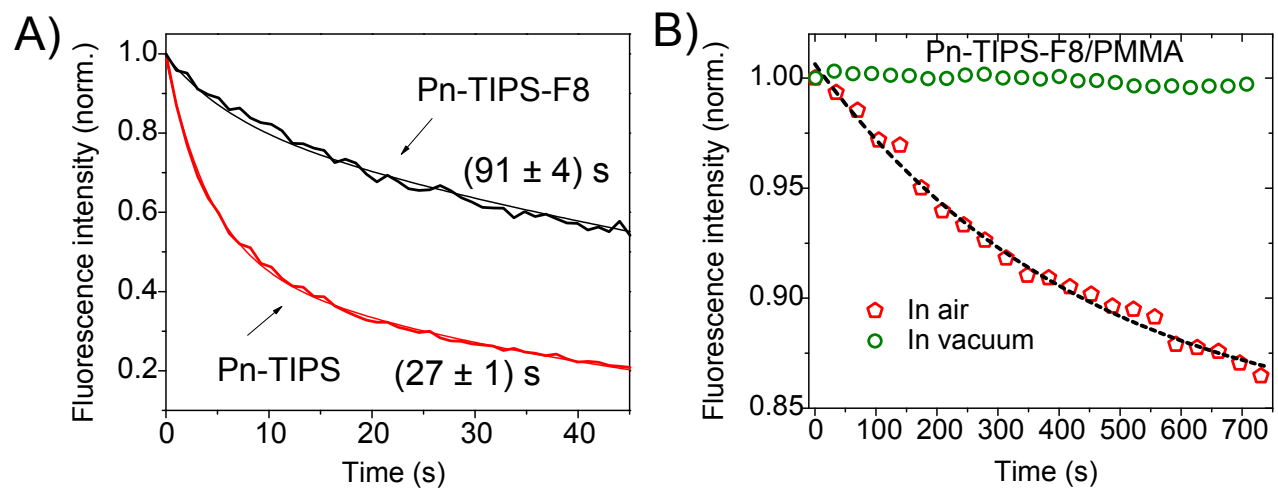

Figure 3. A) Fluorescence decay due to photobleaching under a $633 \mathrm{~nm}$ excitation in a non-fluorinated Pn-TIPS vs a fluorinated Pn-TIPS-F8 derivative in PMMA. Bi-exponential fits and the time constants $\tau_{B}$ are also shown. B) Fluorescence decay in air vs vacuum for Pn-TIPS-F8 in PMMA under $633 \mathrm{~nm}$ illumination.
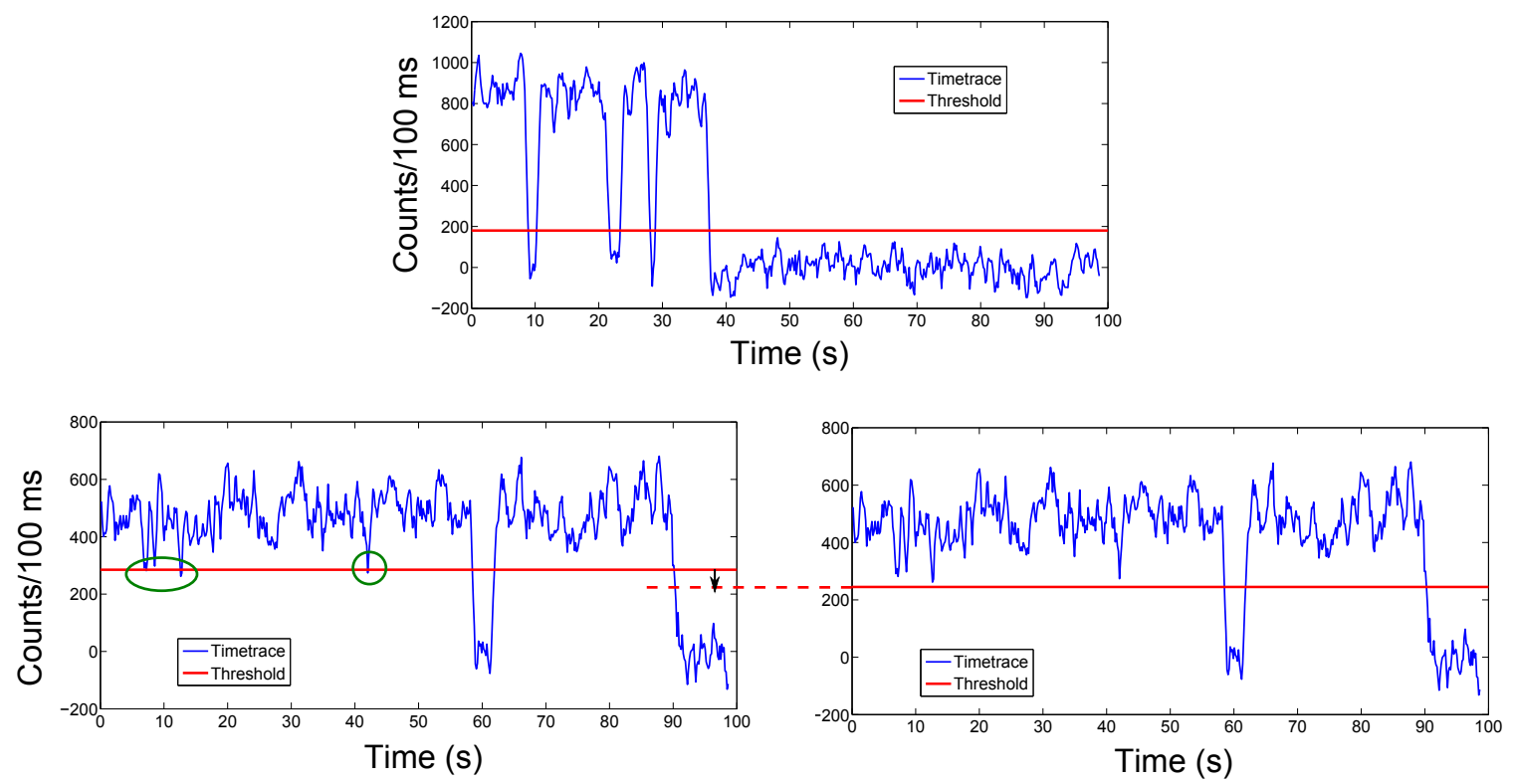

Figure 4. Examples of single-molecule fluorescence time trajectories with various thresholds that separate "on" and "off" states of the molecule. The threshold level was varied to determine how it affects data analysis. Top: Threshold level three standard deviations above the average "off" counts/100 ms. Bottom: Example of threshold that has been lowered such that "on" noise does not interfere with the threshold. 


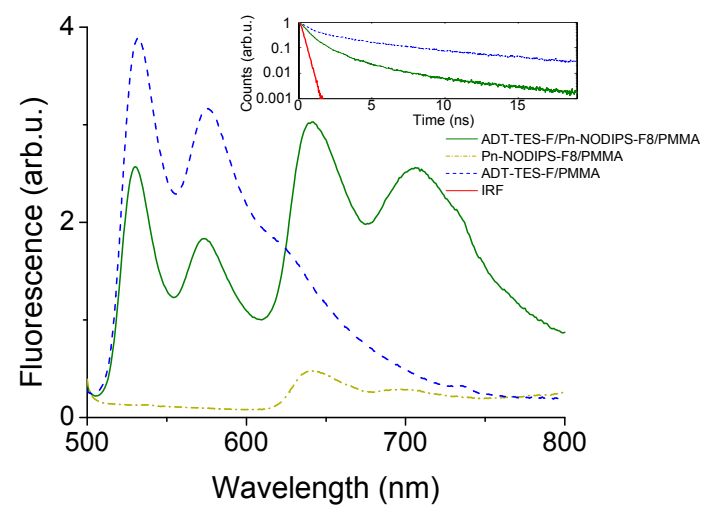

Figure 5. Fluorescence spectra of ADT-TES-F in PMMA (donor-only), Pn-NODIPS-F8 in PMMA (acceptor-only), and ADT-TES-F/Pn-NODIPS-F8 in PMMA (donor/acceptor pair) obtained under $490 \mathrm{~nm}$ excitation. FRET from ADTTES-F to Pn-NODIPS-F8 is observed. Inset shows donor fluorescence lifetime decay obtained under a $532 \mathrm{~nm} 470 \mathrm{ps}$ pulsed excitation of ADT-TES-F in PMMA (donor-only) samples and in ADT-TES-F/Pn-NODIPS-F8 in PMMA (D/A) samples. The instrument response function (IRF) is also included.

radius of $4.8 \mathrm{~nm}$ ) or emissive CT state formation (exciplex) with peak emission at $\sim 669 \mathrm{~nm}$ depending on the spacing between the ADT-TES-F donor and ADT-TIPS-CN acceptor molecules. ${ }^{20}$ Similar trends were observed in ADT-TES-F/Pn-R-F8 blends, in which the emission efficiency and lifetime of exciplex significantly depended on the size of the acceptor's R-group, so that larger $\mathrm{R}$ groups led to a less emissive and shorter lived exciplex. $^{2,5}$ If the ADT-TES-F donor and Pn-R-F8 acceptor molecules are separated by a neutral spacer such as PMMA, FRET, with the FRET radius of 4.2-4.5 nm, depending on the side group $\mathrm{R}$, dominates over the exciplex formation. FRET between ADT-TES-F donor and Pn-NODIPS-F8 acceptor molecules is illustrated in Fig.5 which shows fluorescence spectra from ADT-TES-F donor-only, Pn-NODIPS-F8 acceptor-only, and ADTTES-F/Pn-NODIPS-F8 D/A pair in PMMA under a $490 \mathrm{~nm}$ excitation. In the film containing both donor and acceptor molecules, the fluorescence emission from the acceptor was enhanced due to FRET while the the emission from the donor decreased. The ADT-TES-F donor lifetime also decreased due to FRET (inset of Fig. 5). Our ability to image both donor and acceptor molecules on the single molecule level enables studies which rely on the single-molecule FRET, in various hosts and with an additional benefit of donor and acceptor functionalization flexibility.

\section{CONCLUSIONS}

In summary, we characterized photophysical properties of functionalized anthradithiophene and pentacene derivatives. All molecules studied were imaged on the single-molecule level in polymeric (PMMA, PVK, PS) and crystalline (t-bu BTBTB) hosts using wide-field illumination at room temperature. High fluorescence quantum yields and low photobleaching quantum yields approaching those of standard SMFS fluorophores were obtained. FRET with the FRET radius between 4.2 and $4.8 \mathrm{~nm}$, depending on the acceptor, was observed with the ADTTES-F as the donor and other ADT and Pn derivatives as acceptors. Our studies lay foundations for systematic studies of local environment on photophysics of organic semiconductors at nanoscales and for applications relying on FRET in these materials using SMFS.

\section{ACKNOWLEDGMENTS}

We thank K. Bilty for assistance in imaging of Pn-R-F8 in PVK and PS. This work was supported by the National Science Foundation via CAREER program (DMR-0748671) and grant DMR-1207309.

\section{REFERENCES}

1. O. Ostroverkhova, ed., Handbook of organic materials for optical and (opto)electronic devices, Woodhead Publishing, Cambridge, U.K., 2013. 
2. K. Paudel, B. Johnson, A. Neunzert, M. Thieme, B. Purushothaman, M. M. Payne, J. E. Anthony, and O. Ostroverkhova, "Small-molecule bulk heterojunctions: Distinguishing between effects of energy offsets and molecular packing on optoelectronic properties," Journal of Physical Chemistry C 117, pp. 24752-24760, 2013.

3. A. D. Platt, J. Day, S. Subramanian, J. E. Anthony, and O. Ostroverkhova, "Optical, fluorescent, and (photo)conductive properties of high-performance functionalized pentacene and anthradithiophene derivatives," Journal of Physical Chemistry C 113, pp. 14006-14014, 2009.

4. A. D. Platt, M. J. Kendrick, M. Loth, J. E. Anthony, and O. Ostroverkhova, "Temperature dependence of exciton and charge carrier dynamics in organic thin films," Physical Review B 84, p. 235209, 2011.

5. M. J. Kendrick, A. Neunzert, M. M. Payne, B. Purushothaman, B. D. Rose, J. E. Anthony, M. M. Haley, and O. Ostroverkhova, "Formation of the donor-acceptor charge-transfer exciton and its contribution to charge photogeneration and recombination in small-molecule bulk heterojunctions," Journal of Physical Chemistry C 116, pp. 18108-18116, 2012.

6. J. E. Anthony, "Functionalized acenes and heteroacenes for organic electronics," Chemical Reviews 106, pp. 5028-5048, 2006.

7. O. Ostroverkhova and W. E. Moerner, "Organic photorefractives: Mechanisms, materials and applications," Chemical Reviews 104, pp. 3267-3314, 2004.

8. T. Ameri, P. Khoram, J. Min, and C. J. Brabec, "Organic ternary solar cells: a review," Advanced Materials 25, pp. 4245-4266, 2013.

9. W. E. Moerner, "A dozen years of single-molecule spectroscopy in physics, chemistry, and biophysics," Journal of Physical Chemistry B 106, pp. 910-927, 2002.

10. W. E. Moerner, "New directions in single-molecule imaging and analysis," Proceedings of the National Academy Sciences USA 104, pp. 12596-12602, 2007.

11. W. E. Moerner and M. Orrit, "Illuminating single molecules in condensed matter," Science 283, pp. 16701676, 1999.

12. J. M. Lupton, "Single-molecule spectroscopy for plastic electronics: Materials analysis from the bottom-up," Advanced Materials 22, pp. 1689-1721, 2010.

13. B. Kozankiewicz and M. Orrit, "Single-molecule photophysics, from cryogenic to ambient conditions," Chemical Society Reviews 43, pp. 1029-1043, 2014.

14. J. Bolinger, K. J. Lee, R. Palacios, and P. F. Barbara, "Detailed investigation of light induced charge injection into a single conjugated polymer chain," Journal of Physical Chemistry C 112, pp. 18608-18615, 2008.

15. P. F. Barbara, A. J. Gesquiere, S. J. Park, and Y. J. Lee, "Single-molecule spectroscopy of conjugated polymers," Accounts of Chemical Research 38, pp. 602-610, 2005.

16. S. Park, D. A. Mourey, S. Subramanian, J. E. Anthony, and T. N. Jackson, "High-mobility spin-cast organic thin film transistors," Applied Physics Letters 93, p. 043301, 2008.

17. J. Day, A. D. Platt, O. Ostroverkhova, S. Subramanian, and J. Anthony, "Organic semiconductor composites: influence of additives on the transient photocurrent," Applied Physics Letters 94, p. 013306, 2009.

18. J. Day, A. Platt, S. Subramanian, J. Anthony, and O. Ostroverkhova, "Influence of organic semiconductormetal interfaces on the photoresponse of functionalized anthradithiophene thin films," Journal of Applied Physics 105, p. 103703, 2009.

19. K. R. Rajesh, K. Paudel, B. Johnson, R. Hallani, J. E. Anthony, and O. Ostroverkhova, "Design of organic ternary blends and small-molecule bulk heterojunctions: photophysical considerations," Journal of Photonics for Energy 5, p. 057208, 2015.

20. W. E. B. Shepherd, A. D. Platt, M. J. Kendrick, M. Loth, J. E. Anthony, and O. Ostroverkhova, "Energy transfer and exciplex formation and their impact on exciton and charge carrier dynamics in organic films," Journal of Physical Chemistry Letters 2, pp. 362-366, 2011.

21. K. Paudel, B. Johnson, M. Thieme, M. Haley, M. M. Payne, J. E. Anthony, and O. Ostroverkhova, "Enhanced charge photogeneration promoted by crystallinity in small-molecule donor-acceptor bulk heterojunctions," Applied Physics Letters 105, p. 043301, 2014. 
22. W. E. B. Shepherd, A. D. Platt, M. Loth, J. E. Anthony, and O. Ostroverkhova, "Optical, photoluminescent, and photoconductive properties of functionalized anthradithiophene and benzothiophene derivatives," Proc. of SPIE 7599, p. 75990R, 2010.

23. W. E. B. Shepherd, R. Grollman, A. Robertson, K. Paudel, J. E. Anthony, and O. Ostroverkhova, "Singlemolecule imaging of organic semiconductors: nanoscale insights into photophysics and molecular packing," submitted 2015.

24. W. E. B. Shepherd, A. D. Platt, D. Hofer, O. Ostroverkhova, M. Loth, and J. E. Anthony, "Aggregate formation and its effect on (opto)electronic properties of guest-host organic semiconductors," Applied Physics Letters 97, p. 163303, 2010.

25. T. J. Ha, T. Laurence, D. S. Chemla, and S. Weiss, "Polarization spectroscopy of single fluorescent molecules," Journal of Physical Chemistry B 103, p. 6839, 1999.

26. S. J. Lord, Z. Lu, H. Wang, K. A. Willets, P. J. Schuck, H. D. Lee, S. Y. Nishimura, R. J. Twieg, and W. E. Moerner, "Photophysical properties of acene dcdhf fluorophores: Long-wavelength single-molecule emitters designed for cellular imaging," Journal of Physical Chemistry A 111, pp. 8934-8941, 2007.

27. K. A. Willets, O. Ostroverkhova, M. He, R. J. Twieg, and W. E. Moerner, "Novel fluorophores for singlemolecule imaging," Journal of the American Chemical Society 125, p. 1174, 2003. 